\title{
Scheduled Intravenous Acetaminophen for Postoperative Management of Patients Who Had Thoracoscopic Esophagectomy for Esophageal Cancer
}

\author{
HIROYUKI KITAGAWA ${ }^{1}$, TSUTOMU NAMIKAWA ${ }^{1}$, JUN IWABU $^{1}$, SUNAO UEMURA ${ }^{1}$, MASAYA MUNEKAGE $^{1}$, \\ SACHI TSUDA ${ }^{1}$, KEIICHIRO YOKOTA $^{1}$, MICHIYA KOBAYASHI $^{2}$ and KAZUHIRO HANAZAKI ${ }^{1}$ \\ ${ }^{1}$ Department of Surgery, Kochi Medical School, Kochi, Japan; \\ ${ }^{2}$ Department of Human Health and Medical Sciences, Kochi Medical School, Kochi, Japan
}

\begin{abstract}
Background/Aim: The aim of this study was to assess the effects of scheduled intravenous acetaminophen for postoperative management of patients who underwent thoracoscopic esophagectomy for esophageal cancer. Patients and Methods: The records of 56 consecutive patients who underwent thoracoscopic esophagectomy in the prone position for esophageal cancer were reviewed. For postoperative pain control, twenty-eight patients underwent the scheduled intravenous acetaminophen (SIVA group), whereas the other 28 were managed with intravenous flurbiprofen (Control group). The perioperative outcomes of the two groups were compared. Results: The incidence of pneumonia and the total numerical rating scale of postoperative pain level were significantly lower in the SIVA group than in the Control group (3.6\% vs. $25.0 \%$; $p=0.022$, 40 vs. 93; $p=0.027)$. Patients' fever on the third day after surgery in the SIVA group was significantly lower than in the Control group $\left(36.9^{\circ} \mathrm{C}\right.$ vs. $\left.37.2^{\circ} \mathrm{C} ; \mathrm{p}=0.029\right)$. However, the incidence of anastomotic leakage, laryngeal nerve palsy, the changes of postoperative $C$-reactive protein level, and the duration of hospital stay were not different between the two groups. Conclusion: Scheduled intravenous acetaminophen after thoracoscopic esophagectomy in patients with esophageal cancer was a useful strategy in reducing the incidence of postoperative pneumonia.
\end{abstract}

Esophagectomy with radical lymph node dissection is the main treatment for esophageal cancer, however, it was associated with a high rate of postoperative pulmonary

Correspondence to: Tsutomu Namikawa, Department of Surgery, Kochi Medical School, Kohasu-Okocho, Nankoku, Kochi 7838505, Japan. Tel: +81 888802370, e-mail: tsutomun@kochi-u.ac.jp

Key Words: Scheduled intravenous acetaminophen, thoracoscopic esophagectomy, pneumonia, esophageal cancer. complications (1). To avoid pulmonary complications, postoperative pain control after esophagectomy is important for early recovery, because esophagectomy with two- or threefield lymph node dissection has three incisions (cervical, thoracic, and abdominal) (2), which may affect the patient's postoperative rehabilitation outcome. Less invasive techniques through laparoscopy and thoracoscopy reduce these incisions and patients' postoperative pain. We routinely perform epidural anesthesia to reduce abdominal pain and administer intravenous non-steroidal anti-inflammatory drugs (NSAIDs) when patients complain of pain after esophagectomy.

Recently, the administration of the scheduled intravenous acetaminophen (SIVA) after esophagectomy has been reported to be useful for the patients' early ambulation with a decreased use of opioids (3), which may lower the risk of postoperative bowel palsy and reduce nausea (4). However, few studies have examined the effects of SIVA in reducing the incidence of postoperative pneumonia after thoracoscopic esophagectomy (TSE) in patients with esophageal cancer (5). The aim of this study was to assess whether SIVA reduced the risk of postoperative pneumonia after TSE in patients with esophageal cancer.

\section{Patients and Methods}

SIVA administration for postoperative pain control was used in clinical practice after TSE since January 2015. Before the introduction of the SIVA protocol, postoperative pain control was performed with epidural anesthesia and intravenous flurbiprofen. The data of 60 consecutive patients who underwent TSE for esophageal cancer between October 2013 and October 2017 were reviewed. Two patients who had a history of chemo-radiation therapy, and two who had colon reconstruction were excluded. A total of 56 patients were analyzed.

Postoperative pain control. An anesthesiologist inserted the epidural catheter before starting the TSE and continuously injected the opioid with ropivacain. Epidural anesthesia was not considered for patients who were taking anti-coagulant drugs; instead, analgesia was 
achieved by patient-controlled administration of the opioid. Twentyeight patients were administered SIVA, $1000 \mathrm{mg}$ of acetaminophen every 8 hours, after the TSE until the 3 rd postoperative day (SIVA group). The other 28 were administered intravenously flurbiprofen (Control group).

Outcome parameters. We compared the patients' characteristics, including age, gender, cancer histological type, clinical stage according to TNM classification 7th edition (6), use of neo-adjuvant chemotherapy, use of epidural anesthesia and patient-controlled analgesia (PCA), operative time, intraoperative blood loss volume, time to first flatus and evacuation, use of postoperative intravenous flurbiprofen for 7 days after the surgery, postoperative complications including pneumonia, surgical site infection, anastomotic leakage, and length of postoperative hospitalization, between the two groups. We also compared the pain level with a numerical rating score (NRS) of the first day after the surgery, postoperative $\mathrm{C}$-reactive protein (CRP) changes (before surgery and postoperative days 1,2,3, and 7) and the postoperative body temperatures (postoperative 3 days) between the two groups. Continuous variables were compared by using the Mann-Whitney $U$ test. Qualitative variables were analyzed by Pearson's chi-squared test. $p$-Values $<0.05$ were considered significant. Informed consent was obtained in the form of opt-out on our hospital website. This study was approved from the Institutional Review Board (ERB-103943).

\section{Results}

Table I summarizes the characteristics of patients in the SIVA and Control groups. No patients had a liver failure associated with SIVA. There were no differences in the characteristics of patients, but the use of neo-adjuvant chemotherapy was more prevalent in the SIVA group than in the Control group. Use of the epidural anesthesia or PCA were not different between the two groups. Total operative time and intraoperative blood loss volume were not different between the two groups.

Regarding postoperative complications, the incidence of pneumonia was significantly lower in the SIVA group than in the Control group (3.6\% vs. 25.0\%; $p=0.022)$. The number of patients who needed mechanical ventilation after the surgery due to severe pneumonia in the SIVA group tended to be lower than that in the Control group (1 vs. 3; $p=0.299$ ). Duration until postoperative bowel movement was not different between the two groups. The frequency of required intravenous flurbiprofen for pain in the SIVA group was lower than that in the Control group (Table II).

The peak levels or changes in the postoperative CRP level and duration of hospital stay were not different between the two groups (Figure 1). The third day after the surgery, body temperature was significantly lower in the SIVA group. $\left(36.9^{\circ} \mathrm{C}\right.$ vs. $37.2^{\circ} \mathrm{C} ; p=0.029$; Figure 2$)$. Figure 3 shows the number of patients of the SIVA and Control groups in each NRS. In the SIVA group, sixteen patients had NRS 0, and seven had NRS 1 to 3 (no need of the additional NSAIDs). In contrast, in the Control group, seven had NRS 0, five had
Table I. Comparison of the characteristics between the SIVA and Control groups.

\begin{tabular}{lccc}
\hline & $\begin{array}{c}\text { SIVA } \\
(\mathrm{n}=28)\end{array}$ & $\begin{array}{c}\text { Control } \\
(\mathrm{n}=28)\end{array}$ & p-Value \\
\hline Gender, Male (\%) & $21(75.0)$ & $23(82.1)$ & 0.746 \\
Age, years, median (range) & $67.5(49-82)$ & $65.5(51-81)$ & 0.544 \\
Histology, SCC (\%) & $25(89.3)$ & $24(85.7)$ & 0.413 \\
Stage I, II/III, IV & $15 / 13$ & $10 / 18$ & 0.179 \\
Neoadjuvant chemotherapy & $19(67.9)$ & $27(96.4)$ & 0.005 \\
Epidural anesthesia (\%) & $21(75.0)$ & $24(85.7)$ & 0.313 \\
PCA (\%) & $4(14.3)$ & $5(17.9)$ & 1 \\
Operative time (min) & $609(503-859)$ & $623(473-772)$ & 1 \\
Blood loss (ml) & $170(70-1600)$ & $155(50-950)$ & 0.333 \\
\hline
\end{tabular}

SCC: Squamous cell carcinoma; PCA: patient's-controlled analgesia.

Table II. Comparison of the outcomes between the SIVA and Control groups.

\begin{tabular}{lccc}
\hline & $\begin{array}{c}\text { SIVA } \\
(\mathrm{n}=28)\end{array}$ & $\begin{array}{c}\text { Control } \\
(\mathrm{n}=28)\end{array}$ & $p$-Value \\
\hline Laryngeal nerve palsy (\%) & $11(39.3)$ & $7(25.0)$ & 0.252 \\
Pneumonia (\%) & $1(3.6)$ & $7(25.0)$ & 0.022 \\
Anastomotic leakage (\%) & $4(14.3)$ & $2(7.1)$ & 0.388 \\
Wound infection (\%) & $5(17.9)$ & $4(14.3)$ & 1 \\
Time to first flatus (POD) & $2(1-5)$ & $2(1-6)$ & 0.801 \\
Time to first evacuation (POD) & $5(1-10)$ & $5(2-9)$ & 0.943 \\
Use of NSAIDs (times) & $1(0-7)$ & $3(0-11)$ & 0.003 \\
Hospital stay (days) & $19(13-55)$ & $21(10-138)$ & 0.837 \\
\hline
\end{tabular}

POD: Postoperative days; NSAIDs: non-steroidal anti-inflammatory drugs.

NRS 4 to 6 (needed the additional NSAIDs), and four had NRS 7 to 10 (severe pain). The total NRS was significantly lower in the SIVA group than in the Control (40 vs. 93; $p=0.027$ ). Subgroup analysis, excluding patients who had not receive neoadjuvant chemotherapy indicated that the incidence of pneumonia tended to be lower in the SIVA group than in the Control group (5.3\% vs. $25.9 \% ; p=0.061)$.

\section{Discussion}

This study demonstrated that use of SIVA after TSE in patients with esophageal cancer reduced the incidence of postoperative pneumonia and allowed maintenance of stable postoperative pain control, whereas there was no antiinflammatory effect.

Acetaminophen is commonly used for fever and pain; however, few studies have reported on its postoperative pain control efficacy for digestive surgery (5). Compared to 


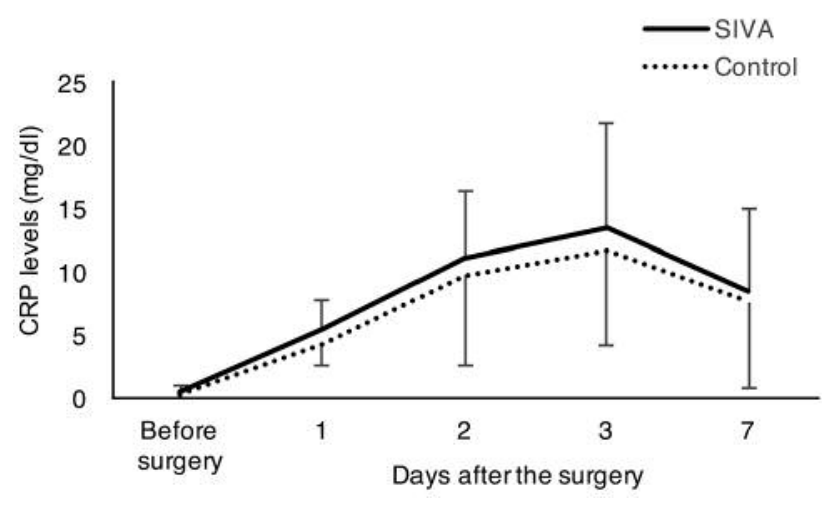

Figure 1. Comparison of the postoperative changes of the C-reactive protein level between the scheduled intravenous acetaminophen (SIVA) and Control groups.

NSAIDs, acetaminophen might be better for postoperative pain management after esophagectomy due to its lower sideeffects induced by cyclooxygenase inhibition, including stomach ulcer, anastomotic leakage, spasm of the respiratory tract, and renal dysfunction (6). On the other hand, acetaminophen has no anti-inflammatory properties. Furthermore, this study demonstrated that there is no difference in the CRP level between the two groups.

We considered that the SIVA protocol lowered the patients' risk of acquiring pneumonia after the TSE by preventing high fever and controlling postoperative pain. Postoperative fever causes increased resting energy expenditure (7) as well as surgical stress (8), and might result in skeletal muscle weakness or pulmonary complications (9). Its pain management that was also contribute to patients' early postoperative ambulation and strong respiration or cough $(10,11)$. These may reduce the incidence of pneumonia without CRP suppression. The number of patients who were administered intravenous NSAIDs was smaller in the SIVA group than in the Control group; fewer intravenous administrations of NSAIDs might be useful in preventing gastric tube mucosal injuries, however, the incidence of anastomotic leakage was not different between the two groups.

This study has certain limitations. This study had a small sample size and a retrospective design. The SIVA group had fewer patients who had received neo-adjuvant chemotherapy. However, in the subgroup analysis, excluding patients who did not receive neoadjuvant chemotherapy, the number of patients who needed mechanical ventilation due to severe pneumonia in the SIVA group tended to be smaller than in the Control group.

In conclusion, SIVA after the TSE in patients with esophageal cancer might be a useful strategy to reduce the

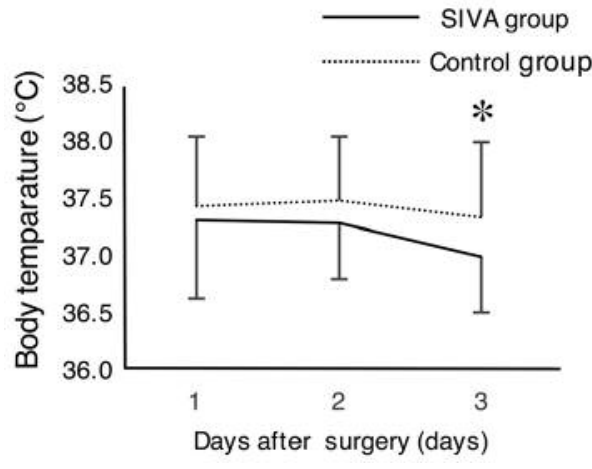

Figure 2. The mean postoperative body temperatures $w 3$ days after the surgery. The third day after the surgery, it was significantly lower in the SIVA group $\left(36.9^{\circ} \mathrm{C} v s .37 .2^{\circ} \mathrm{C} ; p=0.029\right.$, asterisk $)$.

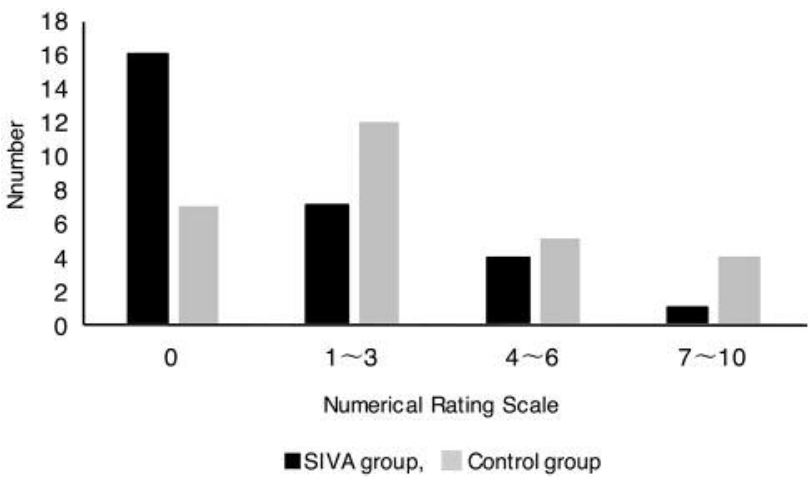

Figure 3. The number of patients of the SIVA and Control group in each numerical rating score (NRS). In the SIVA group, sixteen patients had NRS 0. Whereas, seven in the Control group had NRS 0. There was a significantly difference between the two groups $(p=0.027)$.

incidence of postoperative pneumonia. Further investigations are required to prove the effects of SIVA after TSE, such as comparing the postoperative skeletal muscle mass or resting energy expenditure and conducting a large-scale prospective study.

\section{Conflicts of Interest}

The Authors declare that they have no conflict of interest regarding this study.

\section{Acknowledgements}

The Authors would like to thank Editage (www.editage.jp) for English language editing. 


\section{References}

1 Takeuchi H, Miyata H, Gotoh M, Kitagawa Y, Baba H, Kimura W, Tomita N, Nakagoe T, Shimada M, Sugihara K and Mori M: A risk model for esophagectomy using data of 5354 patients included in a Japanese nationwide web-based database. Ann Surg 260: 259-266, 2014.

2 Nozaki I, Mizusawa J, Kato K, Igaki H, Ito Y, Daiko H, Yano M, Udagawa H, Nakagawa S, Takagi M and Kitagawa Y: Impact of laparoscopy on the prevention of pulmonary complications after thoracoscopic esophagectomy using data from JCOG0502: a prospective multicenter study. Surg Endosc 32: 651-659, 2018.

3 Ohkura Y, Shindoh J, Ueno M, Iizuka T, Haruta S and Udagawa $\mathrm{H}$ : A new postoperative pain management (intravenous acetaminophen: Acelio ${ }^{\circledR}$ ) leads to enhanced recovery after esophagectomy: a propensity score-matched analysis. Surg Today 48: 502-509, 2018.

4 Aryaie AH, Lalezari S, Sergent WK, Puckett Y, Juergens C, Ratermann C and Ogg C: Decreased opioid consumption and enhance recovery with the addition of IV Acetaminophen in colorectal patients: a prospective, multi-institutional, randomized, double-blinded, placebo-controlled study (DOCIVA study). Surg Endosc 32: 3432-3438, 2018.

5 Hughes M, Yim I, Deans DAC, Couper GW, Lamb PJ and Skipworth RJE: Systematic review and meta-analysis of epidural analgesia versus different analgesic regimes following oesophagogastric resection. World J Surg 42: 204-210, 2018.

6 Sobin LH, Gospodarowicz MK and Wittekind C: TNM classification of malignant tumors. 7th ed. Wiley-Blackwell, Oxford, 2010.
7 Bhangu A, Singh P, Fitzgerald JE, Slesser A and Tekkis P: Postoperative nonsteroidal anti-inflammatory drugs and risk of anastomotic leak: meta-analysis of clinical and experimental studies. World J Surg 38: 2247-2257, 2014.

8 Badjatia N, Strongilis E, Prescutti M, Fernandez L, Fernandez A, Buitrago M, Schmidt JM and Mayer SA: Metabolic benefits of surface counter warming during therapeutic temperature modulation. Crit Care Med 37: 1893-1897, 2009.

9 Yatabe T, Kitagawa H, Yamashita K, Hanazaki K and Yokoyama M: Energy expenditure measured using indirect calorimeter after minimally invasive esophagectomy in ventilated postoperative patients. Asia Pac J Clin Nutr 23: 555-559, 2014.

10 Nishigori T, Okabe H, Tanaka E, Tsunoda S, Hisamori S and Sakai Y: Sarcopenia as a predictor of pulmonary complications after esophagectomy for thoracic esophageal cancer. J Surg Oncol 113: 678-684, 2016.

11 Wick EC, Grant MC and Wu CL: Postoperative multimodal analgesia pain management with nonopioid analgesics and techniques: A Review. JAMA Surg 152: 691-697, 2017.

12 Helander EM, Webb MP, Bias M, Whang EE, Kaye AD and Urman RD: A comparison of multimodal analgesic approaches in institutional enhanced recovery after surgery protocols for colorectal surgery: pharmacological agents. J Laparoendosc Adv Surg Tech A 27: 903-908, 2017.

Received November 25, 2018

Revised December 5, 2018

Accepted December 6, 2018 